\title{
Comparison between laparoendoscopic single-site surgery and the conventional total extraperitoneal technique in adult patients for inguinal hernia repair concerning postoperative pain, complications, and cosmesis: A literature review
}

\begin{abstract}
Introduction: Laparoscopic hernioplasty is the gold standard treatment for inguinal hernias if appropriately done. Recently, laparoendoscopic single-site surgery (LESS) has been suggested as an alternative method. However, it is not apparent whether the advantages of this step overcome the possible increased risk of complications such as postoperative pain, recurrence rate, wound infection, seroma, hematoma, and cosmesis.
\end{abstract}

Objective: The aim of this literature review is to compare complications of LESS versus conventional total extraperitoneal (CTEP) technique in adult patients for inguinal hernia repair, in terms of complications.

Method: A search was conducted through Cardiff University databases (PubMed, Scopus, and CINAHL) to find studies that compared and investigated the complications between LESS and CTEP techniques. Seven studies, including five randomized controlled trials (RCTs) and two cohort retrospective studies, were chosen and critically appraised by using books (Moule and Goodman; Grove) and a specific framework, namely the Critical Appraisal Skills Programme (CASP 2017) framework.

Results: There was no significant difference between LESS and CTEP technique in terms of postoperative pain, recurrence rate, wound infection, seroma, hematoma, and cosmesis. However, the cosmetic scar scores were statistically in favour of LESS compared to CTEP groups.

Conclusion: There is still debate in the literature about the superiority of CTEP or the LESS technique. However, more studies are needed to investigate the complications of LESS compared to the CTEP technique in hernioplasty in adult patients.
Volume 6 Issue 4 - 2018

\author{
Ahmed Abdulmohsen Binbosayes \\ Department Specialist / Unit Thrater Manager, Prince Sultan \\ Military Medical City, Saudi Arabia
}

\author{
Correspondence: Ahmed Abdulmohsen Binbosayes, \\ Department Specialist / Unit Thrater Manager, Prince Sultan \\ Military Medical City, Saudi Arabia, \\ Email abinbosayes@gmail.com
}

Received: May 28, 2018 | Published: July 18, 2018

\section{Introduction}

A hernia is an organ's exit into the cavity wall, in which it usually remains, such as the bowel: the word "hernia" comes from the Latin term "rupture". Hernias appear in a number of forms, usually in the abdomen, particularly the groin because the muscles of the abdominal wall weaken with age, ${ }^{1}$ other hernias include hiatus, incisional, umbilical, and femoral hernias. ${ }^{2}$ Tadaki et al. ${ }^{3}$ stated that all abdominal wall hernias detected in the groin are termed 'inguinal', comprising approximately $75 \%$ of hernias.

The inguinal hernia can appear as a lump or swelling in the area of the groin, or as a swollen scrotum. ${ }^{2}$ The lump, which might be painful, usually appears while the patient is lifting anything and disappears while lying down. ${ }^{4}$

An inguinal hernia is located at the pubic bone connecting the lower abdomen and the leg. ${ }^{5}$ It is caused by weakening of the posterior wall, comprised of the conjoint tendon and the transversal is fascia ${ }^{6}$ swelling tissue slides along the canal, which includes the spermatic cord in men and the round ligament in women. ${ }^{2}$

The number of hernia repairs varies among countries: in the United States, it is 28 per 100,000 people, compared to 10 per 100,000 people in the UK. ${ }^{3}$ Ninety-five per cent of patients presenting to primary care are male, and in men the incidence rises from 11 per 10,000 person years aged $16-24$ years to 200 per 10,000 person years aged 75 years or above. ${ }^{7}$

The risk of developing an inguinal hernia is approximately $27 \%$ for males, and $3 \%$ for females, ${ }^{4}$ because of variations in their anatomical structures. ${ }^{8}$ Medically recommend surgery as it can avoid a rare but serious problem named strangulation. This appears once a piece of fatty tissue or a loop of intestine is surrounded in a hernia and the supply of blood is interrupt, which kills the tissue. ${ }^{9}$

One of the most common general surgical conditions, inguinal hernia repairs comprise $7 \%$ of all surgical outpatient procedures performed worldwide every year in the whole population. ${ }^{1}$

Historically, several surgical techniques have been utilized to perform inguinal hernia repairs starting from Eduardo Bassini 1887 by reconstructing the anatomy of the inguinal canal passing through Edward Earl Shouldice 1953, ashis technique called "Shouldice repair" in which the surgeon uses surgical sutures to repair the hernia. ${ }^{10}$ One of the most important methods during this erais Francis Usher 1959 technique by reinforcing Bassini technique with mesh. 
Moreover, the open "tension free" mesh repair technique involves applying a synthetic mesh to close the defect, so that the hernia is repaired without the need to stretch the tissues to meet each other under tension as this method called Lichtenstein technique. ${ }^{11}$ Although these open techniques have been used for decades, many studies have documented that they are associated with high rates of complications, including postoperative pain, hernia recurrence and wound infection. ${ }^{12,13}$ However, laparoscopic hernioplasty methods have also been developed, as well as open tension-free procedures, of which the conventional total extraperitoneal (CTEP) and trans abdominal preperitoneal (TAPP) procedures are the most efficient and frequently used. ${ }^{14}$ For more details, see Appendix A.

Laparoscopic inguinal hernia repair is widely used and has numerous advantages compared to open inguinal hernia repair, such as smaller incisions and less postoperative pain. ${ }^{15,16}$ The other advantages of this type of technique are reduced complications such as recurrence rate, hematoma and seroma, a solid swelling of clotted blood or fluid within the tissues after surgery: Wauschkuhn, and wound infection. ${ }^{17}$

The CTEP inguinal hernia repair regularly needs a total skin incision length comparable to that of an open repair. ${ }^{14}$ Through the surgical procedure, plastic tubes called ports, $5 \mathrm{~mm}$ to $10 \mathrm{~mm}$ in size, are set into these small incisions of up to half an inch. ${ }^{14}$ Laparoscopic instruments and a camera are then entered via the ports, which enable the surgical team to access the inside of the patient. Minimally invasive surgery (Figures 1) (Figure 2) has extended to develop laparoendoscopic single-site surgery (LESS), which can decrease the invasiveness of surgical procedures. ${ }^{18}$

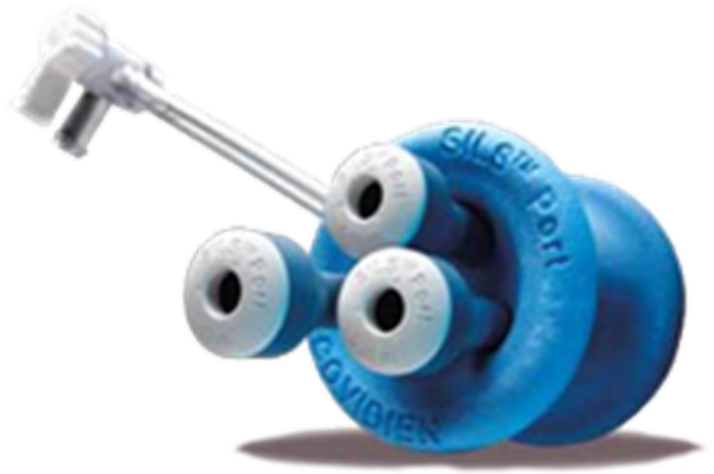

Figure I Photo of Laparoendoscopic single-site (LESS) port.

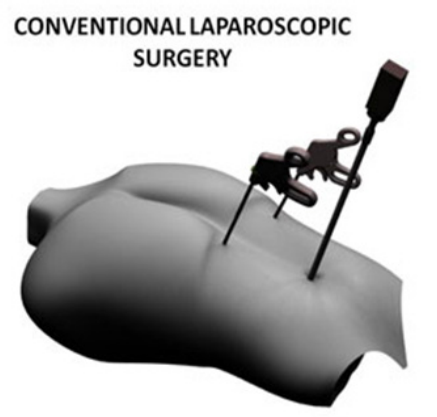
LAPAROENDOSCOPICSINGLE-SITE

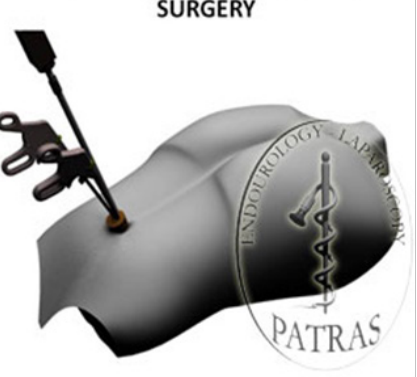

Figure 2 Photo demonstrating the positioning of the single-port with the Laparo-endoscopic single-site approach.

Both CTEP and LESS have provided worthy clinical results in terms of reduced analgesia requirements and superior cosmetic outcomes. ${ }^{19}$
However, there is wide debate surrounding the effectiveness of LESS and CTEP techniques in terms of postoperative pain, hematoma and seroma, wound infection, and cosmetic outcomes..$^{15,16}$ Thus, the aim of this project is to identify and evaluate the relevant evidence on any differences between conventional totally extraperitoneal (CTEP) hernia repair and laparoendoscopic single-site surgery (LESS) in terms of the following outcome measures: postoperative pain, complications such as recurrence rate, wound infections, seroma, hematoma, and cosmetic outcomes.

The objective of this review is to explore the literature and critically discuss relevant evidence to provide meaningful arguments to answer the following question: "In laparoscopic inguinal hernia repair, is the LESS technique better than the CTEP technique in reducing postoperative pain and complications and providing better cosmetic outcomes?"

\section{Methodology}

In order to successfully gain knowledge as to whether the laparoendoscopic single-site (LESS) method could aid in inguinal hernia repair (IHR) in terms of reduced postoperative pain, fewer complications and better cosmetic outcomes compared to the conventional totally extraperitoneal (CTEP) method, this review focuses on recently published studies with appropriate results. Brink state that to enhance practice, seeking evidence on any presented topic and applying such information is paramount to healthcare professionals' development.

Literature reviews, as stated by Gerrish and Lacey, provide benefits in analysing various sources of knowledge efficiently, supporting knowledgeable conclusions. Additional benefits of literature reviews involve: verifying the validity and generalizability of individual studies; recognizing the differences in studies being critiqued and evaluating their accuracy and reliability (Gerrish and Lacey). This methodology is convenient and realistic given the funding available and limited time, and is also cost-effective. ${ }^{20}$ It is hence unsurprising that the Evidence-Based Practice (EBP) action places increasing emphasis on the relevance of literature reviews in healthcare. ${ }^{21}$ The EBP deems literature reviews to be a helpful tool for recognizing and utilizing the findings of the research to obtain practice recommendations, further education and research. ${ }^{22}$ However, the author will select articles which related to the topic and critically appraise the literature review.

\section{Search strategy}

The search for this review was carried out through many different activities, such as electronic databases, books and librarians' opinions. Parahoo stated that utilizing electronic databases enables researchers to obtain published articles immediately and help to ensure that a search is comprehensive. Bowling emphasized that obtaining librarians' guidance is crucial for researchers. ${ }^{23}$ This will ensure that a comprehensive search is achieved, which is vital to form meaningful discussion of the studied problem. ${ }^{24}$ Books and guidelines will be used to get additional information about the literature, as Parahoo states that books can provide extended information which can be used to inform the literature review. To find articles that are relevant to the aim of this literature review, a literature search was carried out electronically within Google Scholar as a scoping exercise. The study was conducted at the researcher's home, because it is a convenient place to study and the Internet is available at all times. 


\section{Search technique}

Initially, to classify a number of articles related to the topic, a scoping exercise was undertaken using Google Scholar. An additional comprehensive search was then conducted using PubMed, Scopus, and CINAHL. These databases were selected for their suitability to address the research question. The three electronic databases were chosen as subject-specific databases, and are all extensive databases that could produce a variety of sources, allowing for a holistic and inclusive literature review. Additionally, the selected databases were used because they provide optimal tools to review literature in the medical field.

\section{Subject identification}

The following keywords were used for the search: "inguinal hernia repair", "inguinal herniorrhaphy", "inguinal hernioplasty", "singleport", "single-incision", "conventional total extraperitoneal (TEP)", "multi-port", "multi-incision", "postoperative pain", "complications", "cosmetic":see Appendix B. By applying the full titles, the following results were found: PubMed yielded four articles resulted (Appendix C), Scopus yielded 27 articles, and CINAHL yielded one article (Appendix D) (Appendix E). LoBiondo-Wood and Haber (2014) stated that the search must utilize phrases or terms that represent each concept of the research. Boolean operators (AND, OR) were utilized to exclude or combine keywords with the research, thus providing more productive and focused results. ${ }^{25}$ This approach preserves effort and time through reducing unsuitable hits that must be checked before discarding.

\section{Selection criteria}

The main purpose of inclusion criteria is to determine the boundaries of the review and to recognise the limitations of the study. ${ }^{23}$ For selecting and accepting articles, therefore, the inclusion criteria that were taken into consideration in this review were as follows:

A. Studies comparing conventional multi-incision and singleincision laparoscopic techniques.

i. Studies focusing on inguinal hernia repair only.

ii. Adult patients (male and female).

B. Studies meet the main outcomes, which are:

i. Postoperative pain.

ii. Complications (recurrence rate, wound infection, seroma, hematoma).

iii. Cosmetic results.

Opinion articles were excluded, as were studies published before 2008, because the LESS approach was first introduced in $2008 .{ }^{26}$ Aveyard states that evidence-based practice should be based on the latest evidence. ${ }^{21}$

Articles must focus on human studies, and must be written in English, which is a scientific language that is universally accepted. Parahoo stated that articles have more credibility if they are published in English.

The application of the abovementioned selection criteria minimized the articles to eleven from the total of 32 articles found.
The full texts of these eleven articles were read to ensure that their outcomes were relevant to the aim of the present study: after reading the full articles, the author chose seven articles which were especially worthy of critical appraisal with regard to answering the question set for this review (Figure 3: PRISMA diagram and Appendix F). Nevertheless, to accommodate a brief and vigorous piece of work, the papers were not reviewed intensely, but with critical judgement, with the review framework in mind. This method, as mentioned by Williamson and Whittaker, is deemed helpful to give a comprehensive literature review while also assuring the validity and value of the presented literature.

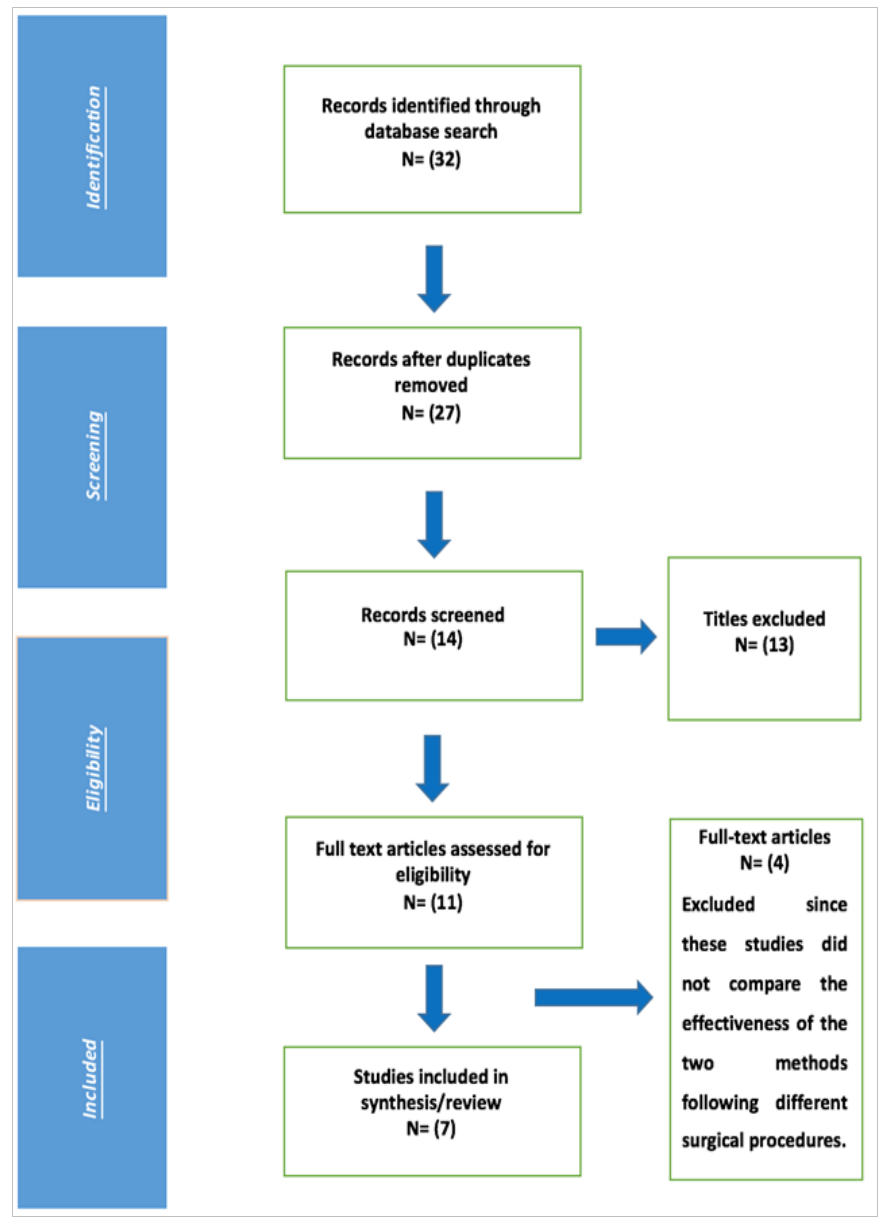

Figure 3 Search and retrieval process.

Five of the chosen studies were RCTs, and two were cohort studies that met the selection criteria (Table 1). RCTs that did not obtain approval from ethical committee and patients' informed consent were excluded. Rees emphasised that ethics is a major consideration in all research stages, as it protects researchers and participants from any potential harm. Additionally, any duplicated published articles were excluded. ${ }^{27}$

As there were many primary studies with different designs investigating the same factors, the author chose to use only studies that give evidence according to the evidence-based practice pyramid (Appendix G), in order to identify optimal approaches and form meaningful arguments from the literature. ${ }^{28}$ 
Table I Summary of Studies Meeting the Search Criteria

\begin{tabular}{llll}
\hline Reference & Origin & Study Design & Sample \\
\hline Wijerathne et al. 2014 & Pubmed/Scopus & Randomised Controlled Trial & I00 Patients \\
Tsai et al. 2013 & Pubmed & Randomised Controlled Trial & 80 Patients \\
Choi et al. 2016 & Scopus/CINHAL & Randomised Controlled Trial & I00 Patients \\
Wijerathne et al. 2016 & Pubmed/Scopus & Randomised Controlled Trial & I00 Patients \\
Buckley et al. 2014 & Scopus/CINHAL & Retrospective Cohort Study & 205 Patients \\
Wakasugi et al. 2015 & Pubmed & Retrospective Cohort Study & I37 Patients \\
Tran et al. 2014 & Pubmed & Randomised Controlled Trial & I00 Pationts
\end{tabular}

\section{Literature review}

\section{Analysis}

The critical appraisal of the literature review will use the framework developed by the Critical Appraisal Skills Programme (CASP 2017: Appendix $\mathrm{H}$ ). The CASP framework gives the needed assistance in analysing research studies in a logical and systemic manner and evaluating the weaknesses and strengths of the methods utilised. ${ }^{23}$ However, not all sub-headings in the framework are discussed due to the restrictions of this dissertation. Additional critique will be based on critical appraisal books such as Parahoo. ${ }^{24,27,28}$

Ethical approval and appropriate authorization were received for all studies used in this literature review. Informed consent signed by patients was provided by all studies' participants, to protect both participants and researchers from harm by maintaining ethical standards. ${ }^{24}$ Moreover, conflicts of interest can occur and might affect the research if researchers have a professional or commercial relationship with another organization or with the people working with them. ${ }^{29}$ In this current case, none of the articles revealed any financial ties or conflicts of interest: this supported the credibility of researchers.

The seven studies identified were allocated to three themes which determine this review outcome. Three studies are critiqued under the first and second themes according to their relationships to the theme's topic, and one study is critiqued in relation to the third theme. Moreover, a discussion will be presented following each theme to simplify the flow of arguments. ${ }^{21}$ The themes for this review were identified as follows:

a. Comparison of LESS with CTEP in terms of postoperative pain assessment.

b. Comparison of LESS with CTEP in terms of complications, such as recurrence rate, wound infection, seroma and haematoma.

c. Comparison of LESS with CTEP in terms of cosmesis.

\section{Postoperative pain}

Laparoscopic inguinal hernia treatment is considered to be the technique of choice to reduce postoperative pain, as surgical incisions are reduced if it is doneproperly, ${ }^{1}$ and postoperative pain intensity and the amounts of analgesics used are lower after laparoscopy, whether using the LESS or the CTEP approach.

Recent studies have compared LESS with conventional laparoscopic treatment. ${ }^{30-32}$ LESS requires one incision, compared with the conventional approach, which required three incisions. Thus, LESS might show superiority over the conventional approach by reducing postoperative pain.

Thus, the following theme will critique three RCTs, all related to postoperative pain in laparoscopic single-site surgery versus conventional total extra peritoneal repair. These articles are as follows:

i. A prospective randomized controlled trial to compare single-port endo-laparoscopic surgery versus conventional TEP inguinal hernia repair. ${ }^{14}$

ii. Laparoendoscopic single-site versus conventional laparoscopic total extra peritoneal hernia repair: a prospective randomized clinical trial. ${ }^{33}$

iii. Single-port versus conventional three-port laparoscopic totally extra peritoneal inguinal hernia repair: a randomized controlled trial. $^{19}$

Wijerathne S, et al. ${ }^{14}$ in Singapore, Tsai YC, et al..$^{33}$ in Taiwan, and Choi BJ, et al. ${ }^{19}$ in Korea, carried out prospective RCTs to compare LESS versus CTEP in adult patients in terms of postoperative pain.

Clear introductions were presented in all articles, reporting the rationale and the hypothesis for conducting the studies, which will have positive impact toward the quality of evidence presented. ${ }^{12} \mathrm{An}$ article's introduction must be clear to the reader and should contain a literature review that explains the points examined in earlier studies and upholds the claim for a new study to clarify the gap in knowledge.

The researchers of the current studies demonstrate what is known and recognise gaps in the present body of knowledge in the final paragraphs of their literature reviews to explain the importance of the research and identify the objectives of the study. ${ }^{29}$ The studies' questionsare clear and suitable, utilizing the Population, Intervention, Comparison, Outcomes (PICO) format. The studies' aimsare clear and objectives are presented at the end of the introductions of all three presented studies, which are to evaluate the superiority of LESS versus CTEP in terms of postoperative pain in adult patients. Parahoo stated that the Introduction should include all relevant information around the research question.

All articles recognise inclusion and exclusion criteria in their method sections: researchers must identify these criteria to enhance the quality of their studies and improve their external validity. ${ }^{25}$ The researchers adequately documented their selection criteria for participants. A comprehensive explanation of eligibility criteria will 
aid in interpreting the finding of the studies and their generalizability. ${ }^{24}$

Tsai et al. ${ }^{33}$ included all kinds of inguinal hernia in their inclusion criteria, such as a recurrent, bilateral and primary hernia. However, Wijerathne et al. ${ }^{14}$ and Choi et al. ${ }^{19}$ excluded recurrent and bilateral inguinal hernias, butdid not declare the reasons behind these exclusions: this might influence the generalizability of the research findings. ${ }^{29}$ Laparoscopy allows for bilateral hernia to be repaired in a single operation without the need for additional ports or incisions. ${ }^{34}$ Also, it allows better identification of femoral hernias, which account for $9 \%$ of recurrent hernias. ${ }^{35}$ The National Institute for Health and Clinical Excellence ${ }^{36}$ recommends the adoption of laparoscopic repair for both recurrent and bilateral inguinal hernias. ${ }^{17}$

The use of suitable instruments for data collection must be justified by the investigator. ${ }^{27}$ To evaluate postoperative pain and to conduct follow-up pain assessment, all articles used a Visual Analogue Scale (VAS) ranging from 0 to $10(0=$ no pain; $10=$ worst possible pain $)$, and classified pain as low (1 to 3 ), moderate (4 to 6 ), and high (7 to 10 ). This kind of pain scale is an appropriate tool to measure the primary endpoint, and the VAS is a particularly reliable and valid instrument, it is easy to develop for researchers and widest possibility range of expression: this increases the reliability of the studies' results. ${ }^{24}$

Before carrying out a study, it must be ensured that the sample size is adequate for cost and ethical purposes. Tsai et al..$^{33}$, Wijerathne et al. ${ }^{14}$, Choi et al. ${ }^{19}$ all enrolled 100 patients, justified by power calculation, which is a step that can be used to ascertain the numbers of participants required to detect an intervention effect significantly. ${ }^{29}$ All articles set their power at $80 \%$ : this means that there is a high chance of detecting a difference between two techniques if it exists. ${ }^{24}$ Justifying their sample size based on power calculations thus increases the generalizability of their findings. ${ }^{29}$

All of researchers in this theme used a proper design that is applicable for their aim, namely the RCT design. An RCT is a type of experimental design and is appropriate if the research question involves a comparison of effects or treatments, ${ }^{24}$ as the participants are divided into groups to overcome confounding variables.

Proper randomization must ensure allocation concealment to avoid selection bias. ${ }^{37}$ In the present RCTs, all participants assigned to the two study arms were equally randomised and divided into two groups $(\mathrm{LESS}=50)$, and $(\mathrm{CTEP}=50)$. For instance, Tsai et al. ${ }^{33}$, Choi et al. ${ }^{19}$ used randomised computer-generated numbers for group allocation: this method of randomization decreases potential bias. They also used sealed envelopes that were allocated by two of the researchers and opened before the procedure: this approach is valid, ${ }^{32}$ and will minimise selection bias and increase the validity of results. ${ }^{24}$ Meanwhile, Wijerathne used the closed envelope method to assign each patient randomly to one or other of the two study arms, which is a suitable method if properly done. However, the researchers failed to state that had completed this randomization or how the process was completed: this will decrease the study's validity and might affect the reliability of the randomization method.

Blinding of investigators increases the reliability and rigour of the results and minimises detection bias; ${ }^{27}$ furthermore, it supports the validity of outcomes and is crucial for the RCT studies. ${ }^{38}$ Detection bias refers to "systematic differences between groups in how outcomes are determined" (Rothman). The double-blind design was used by Wijerathne et al. ${ }^{14}$ and Choi et al. ${ }^{19}$ : this is advantageous, as it guarantees that researchers and participants are blind to allocation and thus decreases the risk of bias and enhances the credibility of results. ${ }^{24}$ In contrast, Tsai et al. ${ }^{33}$ conducted their study as a single blind RCT, as the participants but not the researchers were blinded to the study groups. They did not specify whether their assessors were blinded. This might increase the likelihood of detection bias, and might have an influence on the validity of the results. ${ }^{27}$

Moreover, in all three studies, postoperative pain was evaluated on the ward, and follow-up assessment was carried out in the outpatient clinic. Tsai et al..$^{33}$ and Choi et al. ${ }^{19}$ failed to state who conducted this assessment; hence, their results might be misleading and their accuracy might be decreased. In Wijerathne et al. ${ }^{14}$ the assessment was reported by expert blinded nurses, which helps to enhance the validity of results and decrease selection bias. ${ }^{24}$

The researchers in all three studies under review used several appropriate statistical analyses to analyse the collected data. For instance, the researchers used Fisher's exact test or Chi-squaretest to analyse categorical variables. ${ }^{27}$ For nonparametric data, the MannWhitney U-test was used to identify any significant differences between the LESS and CTEP techniques: this test seems to be appropriate for this purpose. ${ }^{38}$

In all three studies, the researchers calculated P-values indicate the significance of findings. P-values are used to define probability, where $\mathrm{P}<0.05$ is considered as statistically significant. Brown stated that by specifying the significance of results, P-values help to test hypotheses statistically.

Wijerathne et al. ${ }^{14}$, Tsai et al. ${ }^{33}$, Choi et al. ${ }^{19}$ all used figures and tables to display their results appropriately, providing ease of reading (Boswell and Cannon). Moreover, the researchers showed their findings in a clear and understandable way. All three trials were reported in accordance with the Consolidated Standards of Reporting Trials (CONSORT 2010) statement. The CONSORT statement has been adopted by major medical and nursing journals to help readers track study steps. ${ }^{29}$ This will ensure the highest reporting quality.

In the present studies, the researchers explicitly defined their results in numerical and table form in their results sections. This allows readers to evaluate the evidence more easily. Grove stated that this helps readers to determine whether the study results are of use to their current practice.

In Tsai et al. ${ }^{33}$ study, there were no significant differences between the two groups in regard to their baseline characteristics, such as age, gender, American Society of Anaesthesiologists (ASA) status and comorbidity. On the other hand, in Wijerathne et al. ${ }^{14}$ and Choi et al. ${ }^{19}$ studies, all participants were males, but the researchers failed to justify why females were excluded: this might influence the validity of the findings with respect to selection effects (Young and Langford 2013). The exclusion of female patients means that their results cannot be considered as generalizable, while just involving males has little contextual significance. However, as previously mentioned in the introduction, inguinal hernia is more common among adult men $(27 \%$ for malesvs. $3 \%$ for females) because of differences in anatomical structure. Thus, more details should have been given to enhance the studies' generalizability. ${ }^{24}$

Limitations can affect a study's results, and can be defined as research weaknesses. ${ }^{27}$ Evaluation of a study's weaknesses and strengths will assist the reader to determine the significance of its 
results to healthcare practice. Tsai et al. ${ }^{33}$ and Choi et al. ${ }^{19}$ clearly acknowledged their limitations of their studies. However, Wijerathne et al. ${ }^{14}$ study limitations were not clearly written. When judging the validity of their results, researchers should carefully assess the limitations and the strengths of their study. ${ }^{38}$ The discussion should reflect each step of the research process and address potential threats to internal validity or bias and external validity or generalizability (LoBiondo-Wood and Haber).

The researchers illustrated their conclusions in a clear manner, by summarizing what the studies intended to discuss and responding to the research questions: this is essential in any study. Wijerathne et al. ${ }^{14}$ and Choi et al. ${ }^{19}$ concluded that there was no difference between the two arms of laparoscopic techniques in terms of postoperative pain, while Tsai et al. ${ }^{33}$ found that LESS is superior to the CTEP approach. In addition, all discussed RCTs reported CONSORT statements (2010): this is necessary to ensure the reporting quality of RCTs.

\section{Results I}

In Wijerathne et al. ${ }^{14}$ the result of the VAS postoperative evaluation showed no significant difference between the LESS and CTEP arms ( 2.7 vs. 2 , respectively: $\mathrm{p}=0.187$ ). However, in Tsai et al. ${ }^{33}$ the mean pain score at two hours was significantly higher in the CTEP arm than in the LESS arm postoperatively at rest (3.9 vs. 2.6, respectively: $\mathrm{p}=0.02$ ). In Choi et al. ${ }^{19}$ the postoperative pain score at $24 \mathrm{~h}$ showed no significant differences between the two groups (1.4 vs. 2.4 respectively: $\mathrm{p}=0.44$ ) (Figure 4 ).

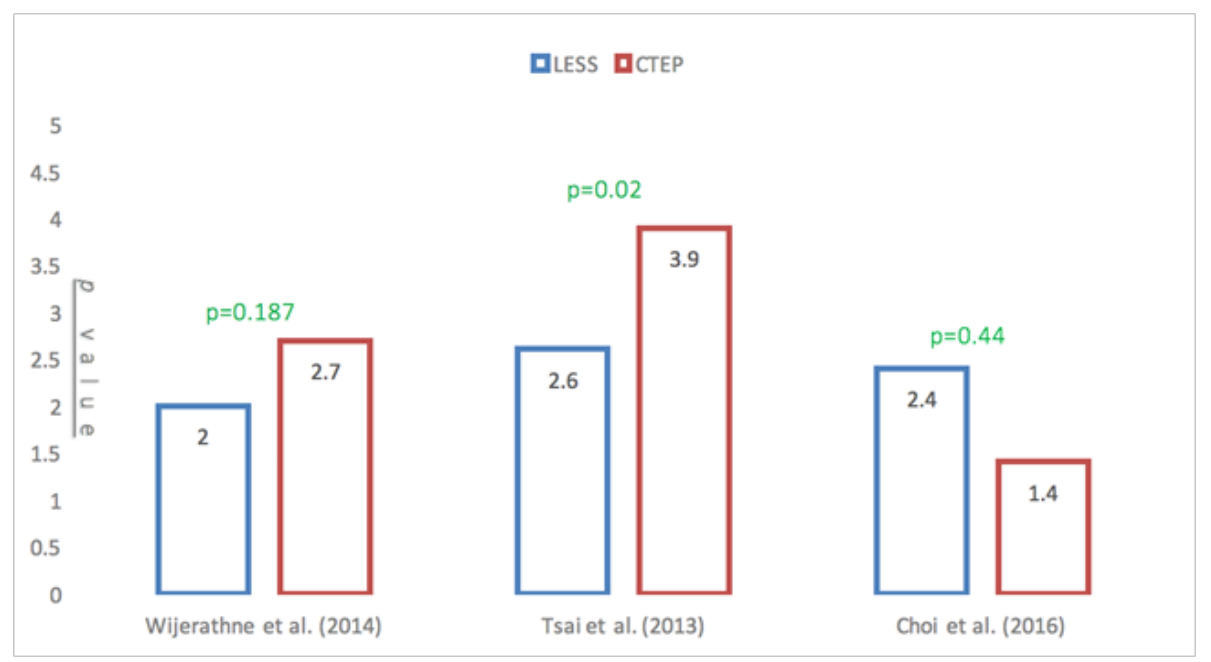

\section{Figure 4 VAS postoperative pain score results.}

Moreover, Wijerathne et al. ${ }^{14}$ and Tsai et al. ${ }^{33}$ assessed postoperative pain after three months for the two arms and found no significant differences: $(\mathrm{p}=0.3)$ in Wijerathne et al. ${ }^{14} ; \mathrm{p}=0.40$ in Tsai et al. ${ }^{33}$. Choi et al. ${ }^{19}$ found no significant differences when they assessed the postoperative pain after four weeks $(\mathrm{p}=0.60)$, but this period seems to be too short, as Weiss et al. ${ }^{39}$ state that pain can persist for six months after the initial repair of inguinal hernia. This influences the validity of the results. ${ }^{40}$

\section{Discussion I}

Postoperative pain is one of the major concerns in inguinal hernia repair, and to date, there is still debate as to whether LESS or CTEP is superior in this respect. ${ }^{1}$ Thus, the discussion in this section is divided into three themes to discuss the findings of Wijerathne et al. ${ }^{14}$, Tsai et al. ${ }^{33}$ and Choi et al. ${ }^{19}$ in a clear manner.

Firstly, there was a contrast in the results of these three studies: for instance, Wijerathne et al. ${ }^{14}$ found no significant difference between the CTEP and LESS techniques in terms of postoperative pain $\left(\mathrm{p}=0.187\right.$ ). Tsai et al. ${ }^{33}$ found that postoperative pain at two hours was significantly higher in the CTEP group than in the LESS group, suggesting the superiority of LESS over the CTEP technique (3.9 vs. 2.6, respectively: $\mathrm{p}=0.02$ ). However, in Choi et al. ${ }^{19}$ there was no significant difference between the two arms after 24hours $(\mathrm{p}=0.44)$, but after one week, pain was significantly lower in the LESS group. Fitzgibbons and Muschalla et al. ${ }^{1}$ expected that because of the reduced number of skin incisions, the LESS method would decrease early postoperative pain. However, Krell et al. ${ }^{41}$ and Weiss et al. ${ }^{39}$ argue that the surgeons' experience might affect the results of single-port surgery. Additionally, Greco et al. (2012) stated that, not surprisingly, the only difference between the LESS and CTEP approachesis the number of skin incisions, as the same surgical techniques are used.

The postoperative follow-up for pain assessment was measured after one month and three months in the three presented RCTs. The results showed no significant difference between groups $(\mathrm{p}=0.333)$, $(\mathrm{p}=0.6)$, and $(\mathrm{p}=0.40)$ respectively.

Secondly, the main reason for the follow-up period is the chronic pain after hernia repair. Wijerathne et al. ${ }^{14}$ and Tsai et al. ${ }^{33}$ assessed pain at three months, while Choi et al. ${ }^{19}$ measured the postoperative pain at one month. All studies seem to need further follow-up pain assessment for at least six months, which might have enhanced the credibility of their results. ${ }^{24}$ Johansen et al ${ }^{42}$ state that strategies for the treatment and prevention of chronic postoperative pain focus on the role of nerve damage. The International Association for the Study of Pain has defined chronic pain as pain lasting more than three months. ${ }^{42}$

Finally, Tsai et al. ${ }^{33}$ compared their results with studies that used the LESS technique in different procedures, such as cholecystectomy, appendectomy and colon resection (Kwon et al. ${ }^{30}$; Tai et al..$^{31}$; Kim et al. ${ }^{32}$ ), perhaps because of the lack of previously published studies on 
inguinal hernia repair. Wijerathne et al..$^{14}$ and Choi et al. ${ }^{19}$ compared their results with recently published studies that compared LESS with CTEP for inguinal hernia repair, as well as studies using different procedures.

Tsai et al. ${ }^{33}$ concluded that LESS has several clinical advantages over CTEP with respect to postoperative pain. ${ }^{43,44}$ In contrast, Wijerathne et al. ${ }^{14}$ and Choi et al. ${ }^{19}$ did not observe any significant differences between the CTEP and LESS groups in the follow-up period.

The significance of the findings was declared in the discussion sections in all articles. These should be accounted for within the overall limitations and strengths of the studies. ${ }^{29}$ Nevertheless, some consideration should be presented as to whether or not the studies findings were externally valid and generalizable. As previously mentioned, Tsai et al. ${ }^{33}$ and Choi et al. ${ }^{9}$ clearly stated their strengths and limitations, whereas Wijerathne et al. ${ }^{14}$ failed to present any limitations, which might affect the generalizability of their study.

\section{Complications}

Laparoscopic treatment is the gold standard in the management of symptomatic inguinal hernia if well done by expert surgeons. ${ }^{32}$ The advantages conferred by minimally invasive surgery involve reduced complications such as recurrence rate, wound infection, seroma and hematoma. ${ }^{45}$ In the quest to make minimally invasive surgery more patient-friendly, with fewer complications, laparoscopic single-site surgery (LESS) has been introduced as a novel technique.

One RCT and two cohort studies will be included in this theme, all related to complications in laparoscopic single-site surgery (LESS) versus the conventional total extra peritoneal (CTEP) approach. These studies are as follows:

i. Single-port versus conventional laparoscopic total extraperitoneal inguinal hernia repair: a prospective, randomized, controlled clinical trial. ${ }^{14}$

ii. The cohort studies are: Wakasugi M. Single-incision totally extra peritoneal inguinal hernia repair: our initial 100 cases and comparison with conventional three-port laparoscopic totally extra peritoneal inguinal hernia repair.

iii. Comparison of outcomes for single-incision laparoscopic inguinal herniorrhaphy and traditional three-port laparoscopic herniorrhaphy at a single institution. ${ }^{46}$

Wijerathne et al. ${ }^{14}$ in Singapore, carried out a prospective randomised trial to compare complications of laparoscopic inguinal hernia repair using either the LESS or the CTEP technique. These complications include recurrence rate, wound infection, seroma, and hematoma. The researchers clearly highlighted the gap in knowledge and presented their aims. ${ }^{24}$ The study's objective was also clearly stated at the end of the introduction. Researchers should define the objective and characteristics of the participants and recognize the population to whom the study's outcomes can be generalized. ${ }^{38}$

Wijerathne et al. ${ }^{14}$ clearly stated their participants' inclusion and exclusion criteria. The inclusion criteria were American Society of Anaesthesiology (ASA) class 1 or 2, aged from 18 to 80 years, and they included male patients only. They excluded patients with bilateral inguinal hernia, obstructions, bleeding disorders and recurrent hernia. Clearly identifying the inclusion and exclusion criteria and explaining the reasons for excluding participants provides greater validity for the study. ${ }^{29}$
The sample size in the study was ninety-nine participants. Wijerathne et al. ${ }^{14}$ performed a power calculation to define the number of participants required in the trial, which was eighty: power calculation is a measure used to determine the number of participants required to detect an intervention effect significantly. In total, 104 patients were enrolled in the trial: twenty-four patients were added in case of dropout. This was sufficient, as four patients declined to participate, and one patient in the LESS group declined to participate after randomization. However, Wijerathne et al. ${ }^{14}$ only recruited involved male patients, as mentioned previously in more detail in theme 3.2. Thus might affect the study's generalizability and might influence the validity of the findings. ${ }^{25}$ Researchers should always clearly explain the reasons behind the exclusion of participants.

Appropriate randomization must certify allocation concealment to minimise selection bias. Wijerathne et al ${ }^{14}$ randomly distributed their participants into two groups $(\mathrm{CTEP}=50$ patients, LESS=49) using a computer-generated statistical tool, which, according to Polit and Beck (2013), is an appropriate method to decrease the risk of selection bias.

Wijerathne et al. ${ }^{14}$ used an identical type of wound dressing as a valid method to blind assessors in both groups. The difference between the two approaches is in the number of surgical incisions: CTEP surgery uses three incisions, while LESS uses just one. The use of the same dressing for all patients thus avoids bias. As emphasised by DiCenso et al ${ }^{47}$ this type of blinding strategy is sufficient in these situations and reduces detection bias by increasing the study's validity.

Mean and standard deviation (SD) were used to express quantitative variables such as age and BMI, which is appropriate (Houser). Wijerathne used SPSS (version 22.0.0.) for statistical analysis. The continuous variables were analysed using t-tests for parametric data and Fisher's exact test for categorical (nominal) variables where appropriate. Thus, the statistical analyses discussed are suitable, as the reliability and validity of the outcomes are reflected by the efficiency of the tools used for the measurement and data collection processes (Ross). For all tests used in the study, the null hypothesis was rejected and the results were deemed statistically significant when the $p$-value $\leq 0.05$, allowing the reader to understand the results' significance and the possibility that they had happened by chance. ${ }^{29}$

Wijerathne displayed their results as figures, charts and tables, thus enabling readers to understand each group's results and the variation between them.

The patients within the research groups in Wijerathne are similar in terms of baseline characteristics. This decreases the chance of confounding variables influencing the outcomes and improves the study's internal validity. ${ }^{29}$

Wijerathne et al. discussion was clear and understandable. The researchers compared their results with those of previous studies. However, they did not acknowledge the study's limitations: LoBiondoWood and Haber stated that researchers should recognize their studies' limitations while reviewing their findings, which contributes to the validity of their results.

Wijerathne et al. conclusion was clear, reasonable and relevant to the results: the researchers concluded that the LESS technique is comparable but not superior to the CTEP technique in terms of complications. As Parahoo stated, to achieve the study requirements, the conclusion must be associated with the results and address the research question or hypothesis. 


\section{Results 2}

During the six months' follow-up of recurrence rate and wound infection, there was no significant difference $(\mathrm{p}=>0.05)$ between the CTEP and LESS groups [3 (6\%) vs. $3(6.1 \%)$ respectively]. Similarly, there were no significant differences $(\mathrm{p}=0.318)$ between the two groups in seroma scores [7 (14\%) vs. $3(6.1 \%)$ respectively].There was also no significant difference $(\mathrm{p}=0.242)$ in terms of haematoma [0 vs. 2 (4.1\%) respectively]: see Figure 5.

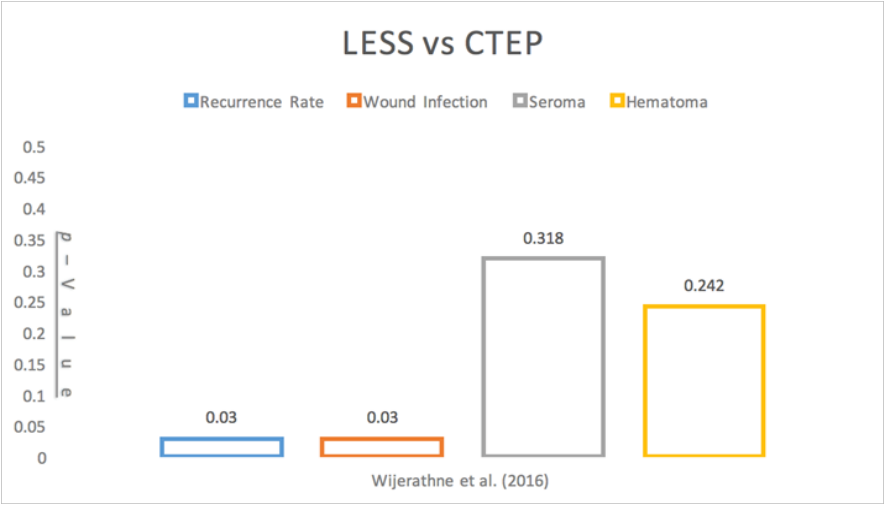

Figure 5 Six-month follow-up complications.

Buckley et al. $^{46}$ in the USA and Wakasugi in Japan carried out cohort studies to compare the LESS and CTEP techniques for inguinal hernia repair in terms of postoperative complications. The researchers of these two studies started their articles by providing satisfactory information in their introductions and clearly stated the aims and objectives, which were based on a justified rationale.

Buckley et $\mathrm{al}^{46}$ and Wakasugi conducted cohort studies, which mainly to test the association between exposure and dependent variables. ${ }^{48}$ The "exposure" in these studies is defined as a factor of interest which may cause or influence an outcome; thus, LESS and CTEP techniques are the exposures and the rate of complications is the outcome of interest. ${ }^{48}$ The dependent variable is defined as what is affected and measured in the experiment. ${ }^{49}$

Buckley et al ${ }^{46}$ and Wakasugi both used a retrospective design. Parahoo states that retrospective studies can produce meaningful results if appropriately conducted. This design has many advantages, including the fact that it typically requires less time to complete, can be carried on a smaller scale, and is useful for investigating various outcomes. ${ }^{24}$ In retrospective studies, the resources tend to be directed at the main collection of data, and such studies are less expensive because the exposure and the outcome have already occurred. Nevertheless, there are disadvantages, including the fact that significant biases might influence the selection of controls, and some relevant statistics cannot be measured ${ }^{50}$ : with retrospective cohort studies, major biases can affect the recall of previous exposure to risk variables. ${ }^{51}$

Mostly, the data recorded in retrospective research have already been recorded for other reasons, such as patient's hospital records (Gearing). In both articles, the researchers used patients' records. However, if data have been recorded in the past, there is commonly a lack of data on possible confounding factors: this is one of the limitations which might affect such studies' validity due to inaccurate and missing data. ${ }^{24}$ Wickson-Griffiths stated that existing records are frequently used for many purposes in health research, such as professional education and assessment of the quality. Moreover, defining the time period is essential in cohort studies to find out how the exposure influenced the outcome of interest within the defined time. ${ }^{24}$ The researchers in both studies clearly defined their time periods: Wakasugi reviewed records from January 2011 to July 2013, and Buckley et al. ${ }^{46}$ reviewed records between 1 August 2008 and 30 July 2012.

Before assembling a cohort study, inclusion and exclusion criteria must be established and an appropriate sample size determined. ${ }^{27}$ Wakasugi and Buckley et al. ${ }^{46}$ clearly defined their selection criteria: for instance, both studies excluded patients who did not receive general anaesthesia, had recurrent, incarcerated or strangulated hernias, and patients with previous lower abdomen surgery. This influences the extent to which the result can be generalized. ${ }^{49}$ Polit and Beck emphasised that identifying inclusion in the study to those with a known diagnosis or specific characteristics, is a powerful strategy to reduce selection bias. ${ }^{29}$

The sampling method chosen in both studies was convenience sampling, which is the most convenient methodology, used in cohort research. Convenience sampling also known as availability sampling "is a specific type of non-probability method that relies on data collection from population members who are conveniently available to participate in study during a time period". ${ }^{52}$

In order to judge whether there is an association between the outcomes and the exposure, sample size must be determined. For instance, Buckley et al ${ }^{46}$ enrolled 205 participants, and compared 129 consecutive patients who underwent LESS with 76 patients who underwent CTEP, whilst Wakasugi investigated a total of 137 patients $($ LESS $=100$; CTEP=37). However, a retrospective cohort study has weaknesses, such as its vulnerability to selection bias. ${ }^{53}$ In both studies, there was no effort to guarantee that patients in the LESS group and the CTEP group presented the same baseline characteristics. This might decrease the studies' generalizability. The sample size in retrospective cohort studies is limited mainly by the availability of patient data in the database. ${ }^{53}$ However, the authors of both articles failed to justify how patients' data were collected and by whom. This may reduce the credibility of the studies, and might decrease their study, as selection bias might be increased. ${ }^{27}$

Wakasugi results are reasonable because there is no difference between the demographic data between patients in the LESS and CTEP groups. In Buckley et al. ${ }^{46}$ hernia characteristics and some demographic data differed between the two arms: for example, recurrent and incarcerated hernias were found in $13 \%$ of patients in the LESS group and $31 \%$ in the CTEP group. This might influence the validity of the results. Overall, in both studies, the researchers answered the research questions made good use of tables and figures and focused on giving written information. They discussed their results in a way that was easy for readers to understand, which is vital.

The discussions in both studies were clear and reasonable, comparing the results with other previous studies. Thus, it is important to compare findings with other related studies; this can set the findings in context and can contribute to readers' knowledge. ${ }^{28}$

Buckley et al. ${ }^{46}$ and Wakasugi et al. (2015) clearly identified their studies' limitations. In the healthcare context, appraisal of a study's weaknesses and strengths will support the reader to ascertain the significance and the validity of its results. ${ }^{37}$ 
Finally, Buckley et $\mathrm{al}^{46}$ and Wakasugi aimed to investigate complications of LESS in inguinal hernia repair. Their findings showed that the expected results for CTEP repair of inguinal hernias could be reproduced using the LESS approach if performed by surgeons who are experts in laparoscopic techniques.

\section{Results 3}

During the six-month follow-up period, Buckley et al. ${ }^{46}$ found a recurrence rate of $2.3 \%$ ( 3 of 129) for LESS and $1.4 \%$ (1 of 76 ) for CTEP. With regard to wound complications, both LESS and CTEP resulted in one recorded wound infection. The incidences of seroma and hematoma were not significantly different between the two arms, and the complication rate between the LESS and CTEP did not differ significantly $(\mathrm{p}=0.84)$. Similar findings were also recorded by Wakasugi et al. (2015): there were no significant differences between the two groups with regard to hernia recurrence $(\mathrm{p}=0.3)$. No records were shown for either LESS or CTEP with regard to wound infection during the six-month follow-up period $(\mathrm{p}=0.3)$. Seroma was seen in $2 \%(1 / 37)$ of the patients in the CTEP group and $11 \%(11 / 100)$ of those in the LESS group $(\mathrm{p}=0.2)$. Hematoma was seen in $2 \%(1 / 37)$ of the patients in the LESS group and $0 \%(0 / 100)$ in the CTEP group $(\mathrm{p}=0.3)$ : see Figure 6

\section{LESS vs CTEP}

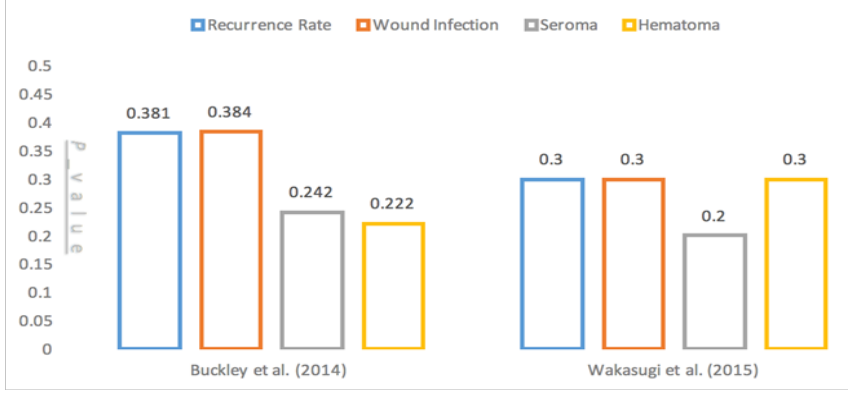

Figure 6 Six-month follow-up: complications.

\section{Discussion 2}

The turnover from CTEP to the LESS technique for inguinal hernia repair has become a smooth and steady journey, following early debate about complication rates. ${ }^{26,39}$ In the quest for scarless surgery and abolition or reduction of parietal trauma, LESS has been touted as the ultimate in surgical innovation for abdominal/pelvic procedures. $^{32,54}$ The three studies reviewed above ${ }^{14,46}$ investigated the rate of complications associated with the two techniques. The discussion in this section is divided into three themes.

Firstly, the results of the current studies showed that there are no significant differences between LESS and CTEP in terms of complications such as recurrence, wound infection, seroma, and hematoma at six-month follow-up. For instance, Wijerathne et al. ${ }^{14}$ results did not show a significant association between recurrence rate and wound infection $(\mathrm{p}=>0.05)$, seroma $(\mathrm{p}=0.38)$, or hematoma $(\mathrm{p}=0.242)$. In Buckley et al. ${ }^{46}$ the complication rate between the two arms did not differ significantly $(\mathrm{p}=0.84)$. Additionally, Wakasugi et al. ${ }^{14}$ found similar results, with no significant difference in the complications in terms of recurrence rate $(\mathrm{p}=0.3)$, wound infection $(\mathrm{p}=0.3)$, seroma $(\mathrm{p}=0.2)$, and hematoma $(\mathrm{p}=0.3)$.

Secondly, the three sets of researchers compared their result with other studies that compared complications between the LESS and CTEP approaches. The majority of the evidence was taken from previous studies involving laparoscopic procedures for appendectomy, cholecystectomy, and partial colectomy, because of the shortage of studies in inguinal hernia procedures. Although the other laparoscopic procedures were conducted in a few small RCTs and large case series, the researchers explained that the LESS approach is similar to the conventional laparoscopic approach in terms of complication rates. ${ }^{55,56}$

Finally, complications might be reduced by surgeons with more experienced hands in both the LESS and the CTEP technique. ${ }^{57}$ According to the Royal College of Surgeons (2016), laparoscopic inguinal hernia repair has been reported to result in fewer complications than open repair if performed by expert hands. Thus, further training and proper education are needed for novel techniques such LESS. As the three studies reviewed in this section ${ }^{14,46}$ involved expert surgeons for both techniques, this will increase the validity of their results .

\section{Cosmetic outcomes}

Since the advent of laparoscopic surgery in the early 1990s, it has been progressively favoured by surgeons and patients worldwide because of its various advantages in terms of patient satisfaction and operative outcomes..$^{58}$ The conventional total extraperitoneal (CTEP) and laparoscopic single-site surgery (LESS) methods have a positive effect on cosmetic outcomes because they involve fewer incisions. Jolley et al. (2013) stated that patients consider the number of scars to be a substantial factor in their cosmetic satisfaction and hence favour LESS rather than the CTEP approach. This factor has contributed to the widespread use of the LESS technique since it was first performed in 2008 by Filipovic-Cugura. ${ }^{59}$

One RCT will be included in this theme, which examines cosmetic outcomes in LESS compared to CTEP. ${ }^{26}$ Potential benefits of singleport compared to multiport laparoscopic inguinal herniorrhaphy: a prospective randomized controlled study.

Tran et al. ${ }^{26}$ in Australia conducted their research as a prospective RCT to evaluate cosmetic outcomes of the LESS technique compared to the CTEP technique in adult inguinal hernia repair. The article starting with an adequate literature review that relates to the research object. Rees ${ }^{27}$ stated that the purpose of a literature review is to present a summary of the presently identified problems or the situation and to recognise gaps in knowledge.

The research's aim and rationale were clearly identified. As defined by Parahoo (2014), the literature review should be a review of any published studies on the subject of interest, with the aim being to clarify the project and the study aim.

Tran et al. ${ }^{26}$ chose a good and reliable design for their randomised trial. Their study was designed according to the CONSORT (2010) statement, as discussed in more detail in theme 3.2. Moher et al. ${ }^{37}$ stated that the CONSORT statement was introduced worldwide to develop the reporting of RCTs, since the evidence has determined that the quality of reporting of RCTs is not optimum.

The researchers evaluated surgical scars after six months using the Patient and Observer Scar Assessment Scale (POSAS), which is valid and reliable: ${ }^{60}$ see Appendix I. Neligan ${ }^{61}$ emphasised that the POSAS has the advantage of combining the patient's opinion and can properly evaluate symptoms such as itchiness, thickness, and pain.

Tran et al. ${ }^{26}$ justified their sample size based on a power calculation, which indicated that the sample should include eighty participants (forty in each group); however, ten more participants were added to 
each group (total of fifty participants) in case of dropouts. Ninety patients who were scheduled for inguinal hernia repair were enrolled in the study, presented an $80 \%$ of power at a significance level of 0.05 . Greenhalgh $^{38}$ stated that expanding the sample size will increase a study's reliability and validity, and is recommended to avoid attrition bias.

Tran et al. ${ }^{26}$ used a computer-generated list of random numbers to allocate their participants randomly into two groups: LESS $(\mathrm{n}=51)$ and CTEP $(\mathrm{n}=49)$. Randomization strengthens a study, ${ }^{27}$ and this allocation strategy decreases the chance of selection bias. ${ }^{38}$

The researchers used suitable statistical tests, such as the MannWhitney U-test for non-parametric continuous variables. Values of $\mathrm{p}<0.05$ were treated as statistically significant. The tools used appear suitable and reliable, and thus increase the validity of the results. ${ }^{20}$

Tran et al. ${ }^{26}$ presented their results in numerical form, in a style that seems clear and well-organized. The researchers explained how these results were determined by applying figures and tables to show their outcomes in a comprehensible and readable way. Presenting results in tabular and numeric form will aid the reader's understanding. ${ }^{62}$ It is clear that the outcomes are related to the research aim and verify the hypothesis.

The study groups were treated equally, as the researchers adopted the same expert surgeon for all surgeries. This will minimise the chance of performance bias by limiting issues that might influence the results.

Tran et al. ${ }^{26}$ discussed their results, acknowledged limitations and compared their findings with earlier studies. Tran et al. ${ }^{26}$ acknowledge their limitations, such as their use of a single surgeon with the potential for bias in favour of LESS and their relatively short follow-up time.

Tran et al. ${ }^{26}$ concluded that such encouraging results were achieved by a dedicated high-volume hernia surgeon, and might not be readily translated into the general surgical community. Therefore, widespread adoption of the LESS technique should await confirmation of its benefits compared to the CTEP technique by large multi-centre, prospective randomized trials. Overall, the methodology applied in Tran et al. ${ }^{26}$ study appears to be valid and sufficient.

\section{Results 4}

The median cosmetic scar scores were statistically in favour of LESS compared to the CTEP group, at 24 (IR 1.0) vs. 21 (IR 2.5), respectively $(\mathrm{P}<0.001)$ : Figure 7 .

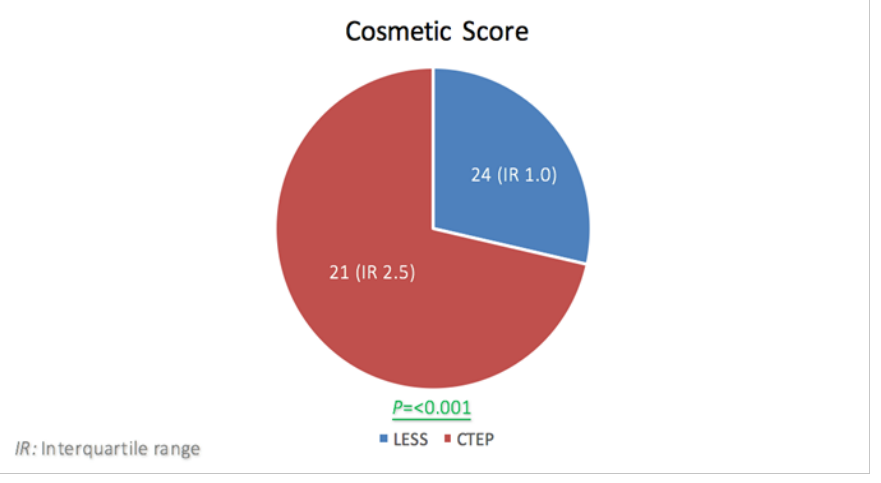

Figure 7 Cosmetic scar score after one-month follow-up.

\section{Discussion 3}

Laparoscopic single-site surgery (LESS) is a recent technique in laparoscopic surgery (Jolley). In this technique, surgery is done using a single incision, generally through the patient's navel. Further, in this kind of surgery, only a single small scar is left behind, rather than the conventional total extraperitoneal (CTEP) approach, which left three scars. Tran et al. ${ }^{26}$ conducted a study to determine which technique is better in terms of cosmesis, as discussed in this section, which is divided into three themes.

Firstly, Ahmed and Paraskeva ${ }^{63}$ and Do et al. ${ }^{64}$ found worthy clinical results in terms of better cosmesis following the LESS technique for laparoscopic inguinal hernia repair, which is quickly taking over from CTEP. The LESS represents a step forward, with fewer surgical incisions but the same benefits. Inguinal hernia may be an especially good application for LESS, as it is a common operation in the younger demographic: a group that places more emphasis on cosmetic outcomes. ${ }^{43}$

Secondly, the researchers compared their results to those of other studies, thus increasing the validity of their outcomes. Four studies have compared the cosmetic results between LESS and CTEP for inguinal hernia repair: two studies found no significant difference. ${ }^{31,33}$ Superior cosmetic results from LESS were reported in one study, ${ }^{64} 5$ and the other two studies found that wounds in the LESS arm were less noticeable to others compared to wounds in the CTEP arm, but that overall, in terms of discomfort, patients from both arms were satisfied. ${ }^{16}$ However, the statistically significant advantage of LESS in cosmetic terms did not translate into any significant differences in quality of life. ${ }^{66}$

The last theme addressed is that hernia repair is a mainstay of general surgery, and that the LESS approach stands to obtain much favour with patients, particularly as demand for increased cosmetic results increases. ${ }^{35}$ Golkar et al. ${ }^{20}$ argued that cosmetic outcomes have not been shown to be consistently better with LESS, and this has, in fact, been shown to be the least important factor for patients contemplating surgery. Choi et al. ${ }^{19}$ reported that postoperatively in the LESS group in their study, all participants indicated that they would stay with their LESS choice, while about $60 \%$ of those in the CTEP group indicated that they would prefer the LESS approach. Thus, patients seemed to prefer the LESS technique when similar surgical outcomes were anticipated. Tran et al ${ }^{26}$ showed that cosmetic scar scores were statistically in favour of LESS compared to the CTEP group ( $\mathrm{P}<0.001)$. However, the lack of studies on cosmetic outcomes has led to debate about which technique is superior. ${ }^{19}$

\section{Discussion of findings}

The laparoscopic technique for inguinal hernia repair was first defined in the 1990s, and underwent continuous development until 2007 when the laparoscopic single-site surgery (LESS) approach was introduced. ${ }^{67,68}$ Safety is always one of the main factors, combined with complication rates. The major advantages of laparoscopic surgery over open surgery - either LESS or conventional total extraperitoneal (CTEP)- are less postoperative pain, fewer complications, and better cosmetic results. ${ }^{67,69,70}$ The studies examined in this review ${ }^{14,46,33,19,26}$ compared LESS with CTEP in terms of postoperative pain, complications such as recurrence, wound infection, seroma and hematoma, and cosmetic outcomes. This section will be divided into three themes in which the seven articles' findings will be discussed.

Citation: Binbosayes AA. Comparison between laparoendoscopic single-site surgery and the conventional total extraperitoneal technique in adult patients for inguinal hernia repair concerning postoperative pain, complications, and cosmesis:A literature review. MOJ Surg. 20I8;6(4):99-II2. 
Firstly, various attempts have been made to minimize postoperative pain following laparoscopy. The LESS technique requires fewer incisions than CTEP. Importantly, LESS is regularly applied in the umbilical area, where there are no muscles, so this approach reduces abdominal muscles damage and the correlated postoperative pain. ${ }^{71,72}$ Studies of postoperative pain reduction in LESS in comparison with CTEP have been performed in various surgical fields, but the findings are still debated. However, Wijerathne et al. ${ }^{14}$; Tsai et al. ${ }^{33}$; Choi et al. ${ }^{19}$ conducted studies to evaluate postoperative pain scores and follow-up assessment for postoperative pain.

Postoperative pain scores were measured at the hospital in the two studies, which found no significant difference between the LESS and CTEP groups ( $\mathrm{p}=0.187$ and $\mathrm{p}=0.44$ respectively). However, Tsai et al. ${ }^{33}$ results revealed significantly higher pain scores in the CTEP group than in the LESS group at two-hour assessment (3.9 vs. 2.6: $\mathrm{p}=0.02$ ). However, postoperative pain assessment at three months revealed no significant differences between the two groups: $(\mathrm{p}=0.333$ in Wijerathne et al. ${ }^{14} \mathrm{p}=0.40$ in Tsai et al. ${ }^{33}$ and $(\mathrm{p}=0.6)$ in Choi et al. ${ }^{19}$ after four weeks.

Secondly, Wijerathne et al. ${ }^{14}$; Buckley et al. ${ }^{46}$ investigated the complications of LESS compared with CTEP in terms of recurrence rate, wound infection, seroma and hematoma. The risk of complications was low, since both approaches were performed by expert laparoscopic surgeons ${ }^{57}$ in all three studies. Overall, there was no significant difference between the two techniques. In Wijerathne et al. ${ }^{14}$ recurrence rate, wound infection, seroma and haematoma showed no significant difference ( $\mathrm{p}=>0.05$ for all measures). Wakasugi et al. ${ }^{14}$ found that the complication rate did not differ significantly between LESS and CTEP when Fisher's exact test was conducted $(p=0.84)$. Lastly, Buckley et al ${ }^{46}$ found no significant difference between the LESS and CTEP groups in terms of recurrence rate $(p=0.3)$, wound infection $(\mathrm{p}=0.3)$, seroma $(\mathrm{p}=0.2)$, and hematoma $(\mathrm{p}=0.3)$.

Finally, the LESS method yields obvious visible cosmetic benefits, due to the smaller number of surgical incisions. The operation is performed within the umbilicus, resulting in hidden surgical wound in the umbilicus. ${ }^{73}$ CTEP method also involves small surgical incisions compared to those from open surgery. Tran et al. ${ }^{26}$ conducted a study to assess the differences in cosmetic scar scores between LESS and CTEP groups. The median scores were statistically in favour of LESS compared to the CTEP group, at 24 (IR 1.0) vs. 21 (IR 2.5): $\mathrm{P}<0.001$.

To date, several studies have demonstrated the clinical safety and technical feasibility of LESS in cholecystectomy, ${ }^{74}$ appendectomy, ${ }^{75}$ and colectomy. ${ }^{76}$ However, limited data are available to compare LESS with the CTEP technique for inguinal hernia repair in terms of complication rates and cosmetic outcomes. ${ }^{77-80}$

\section{Limitations}

There are several limitations to the studies reviewed. For example, the extreme proximity of instruments and the counterintuitive nature of the LESS approach have caused difficulties with the expansion of this novel technique. ${ }^{15,80,81}$

The LESS technique was developed to decrease the invasiveness of conventional laparoscopy and to enhance the cosmetic results. ${ }^{26}$ However, given the short-term durations of the studies reviewed here, it is necessary to conduct studies with long-term follow-up, which will enable consistent quantification of short-term variations and analysis of long-term outcomes. The studies included which related to postoperative pain had short follow-up periods: ideally, the follow-up length needs to be longer than six months, as discussed previously in theme $3.2 .^{82-86}$

Finally, the chosen articles were analysed and appraised by a single novice researcher, based on his limited knowledge, and this should be recognized and acknowledged as a limitation. Therefore, the classification, critical appraisal, and discussion might not be as precise as those achieved by a researcher with more experience. ${ }^{21,86-92}$

\section{Conclusion}

Having critically appraised the selected seven articles to explore the improvement of practice, it can be concluded that the LESS approach is safe and feasible in certain patients in comparison to CTEP and provides comparable results. Further high-powered randomized trials are required to define whether LESS gives any advantages; these further studies should concentrate especially on complication rates, technique failure, analgesia requirements, enhanced recovery after surgery (ERAS), cosmesis and quality of life, and must be conducted by expert hands. It is noteworthy that NICE has not yet published guidelines for the LESS technique.

\section{Recommendations}

After critically appraising and evaluating the seven articles discussed previously, this review concludes that several recommendations might enhance the results of comparison in future. The recommendations are as follows: the issue of complication rates and chronic pain following inguinal hernia repair should be addressed in prospective future studies, as suggested by the National Institute for Health and Care Excellent (NICE 2004) utilizing the standard definitions (acute and chronic) to allow assessment of the degree of pain. Acute pain, such as postoperative pain, subsides as healing takes place, whereas chronic pain is persistent.

The LESS or CTEP for inguinal hernia repair should be carried out only by surgeons who have received proper training and who perform the procedure regularly. As recommended by NICE (2004), laparoscopic surgery as a repair option for inguinal hernia can yield meaningful outcomes, especially if the surgeons have clinical experience. $^{57}$

\section{Acknowledgements}

None.

\section{Conflict of interest}

Author declares there is no conflict of interest in publishing the article.

\section{References}

1. Muschalla F, Schwarz J, Bittner R. Effectivity of laparoscopic inguinal hernia repair (TAPP) in daily clinical practice: early and long-term result. Surg Endosc. 2016;30(11):4985-4994.

2. Kingsnorth A, LeBlanc K. Hernias: inguinal and incisional. Lancet. 2003;362(9395):1561-1571.

3. Tadaki C, Lomelin D, Simorov A, et al. Perioperative outcomes and costs of laparoscopic versus open inguinal hernia repair. Hernia, 2016;20(3):399-404

4. Kavic SM. Laparoscopic versus open repair: a superior approach to inguinal herniorrhaphy. OA Min Inv Surg. 2013;1(1):1. 
5. Garden J, Bradbury A, Forsythe J, et al. Principles and Practice of Surgery. $6^{\text {th }}$ ed. Philadelphia: Churchill Livingstone: Elsevier; 2012.

6. Kontoyannis A, Sweetland H. Crash Course Surgery, $3^{\text {rd }}$ ed. Philadelphia: Churchill Livingstone: Elsevier; 2008.

7. Jenkins JT, O’DwyerPJ. Inguinal hernias. BMJ. 2008;336(7638):269-272.

8. Ruhl CE, Everhart JE. Risk factors for inguinal hernia among adults in the US population. American Journal of Epidemiology 2007;165(10):1154-1161.

9. Bittner R, Montgomery MA, Arregui E, et al. Update of guidelines on laparoscopic (TAPP) and endoscopic (TEP) treatment of inguinal hernia (International Endohernia Society). Surg Endosc. 2015;29(2):289-321.

10. Tolver MA. Early clinical outcomes following laparoscopic inguinal hernia repair. Dan Med J. 2013;60(7):B4672.

11. McLatchie G. Oxford handbook of clinical surgery. $4^{\text {th }}$ ed. Oxford: Oxford University Press; 2013.

12. Gause CD, Casamassima MGS, Yang J, et al. Laparoscopic versus open inguinal hernia repair in children $\leq 3$ : a randomized controlled trial. Pediatr Surg Int. 2016;33(3):367-376.

13. Cavazzola LT, Rosen MJ. Laparoscopic versus open inguinal hernia repair. Surgical Clinics of North America 2013;93(5):1269-79.

14. Wijerathne S, Agarwal N, Ramzy A, et al. A prospective randomized controlled trial to compare single-port endo-laparoscopic surgery versus conventional TEP inguinal hernia repair. Surg Endosc. 2014;28(11):3053-3058.

15. Saha N, Biswas I, Rahman MA, et al. Surgical outcome of laparoscopic and open surgery of pediatric inguinal hernia. Mymensingh Med J. 2013;22(2):232-236.

16. Yang C, Zhang H, Pu J, et al. Laparoscopic vs open herniorrhaphy in the management of Pediatric inguinal hernia: a systemic review and metaanalysis. J Pediatr Surg. 2011;46(9):1824-1834.

17. Karthikesalingam A, Markar SR, Holt PJ, et al. Meta $\square$ analysis of randomized controlled trials comparing laparoscopic with open mesh repair of recurrent inguinal hernia. Br J Surg. 2010;97(1):4-11.

18. Sajid MS, Khawaja AH, Sayegh M, et al. A systematic review comparing single-incision versus multi-incision laparoscopic surgery for inguinal hernia repair with mesh. Int J Surg. 2016;29:25-35.

19. Choi BJ, Jeong WJ, Lee IK, et al. Single-port versus conventional three-port laparoscopic totally extraperitoneal inguinal hernia repair: a randomized controlled trial. Hernia. 2016;20(6):789-95.

20. Golkar FC, Ross SB, Sperry S, et al. Patients' perceptions of laparoendoscopic single-site surgery: the cosmetic effect. $A m J$ Surg. 2012;204(5):751-61

21. Aveyard H, Sharp P. A beginner's guide to evidence-based practice in health and social care. $2^{\text {nd }}$ ed. Milton Keynes: Open University Press; 2013.

22. Fulton S, Krainovich-Miller B. Learninga outcomes. Nursing Research: Methods and Critical Appraisal for Evidence-Based Practice. 2014. 49 p.

23. Bowling A. Research methods in health: investigating health and health services. $4^{\text {th }}$ ed. Maidenhead: Open University Press; 2014.

24. Moule P, Goodman M. Nursing research: an introduction. $2^{\text {nd }}$ ed. London: Sage Publications; 2013.

25. Young A, Langford RH. Making a difference with nursing research. Pearson Higher; 2013.
26. Tran H, Turingan I, Tran K, et al. Potential benefits of single-port compared to multiport laparoscopic inguinal herniorrhaphy: a prospective randomized controlled study. Hernia. 2014;18(5):731-744.

27. Rees C. An introduction to research for midwives. $3^{\text {rd }}$ ed. Elsevier Health Sciences; 2012.

28. Burns N, Grove SK. Understanding nursing research: Building an evidence-based practice. Texas: Elsevier Health Sciences; 2010.

29. Polit DF, Beck CT. Nursing research: generating and assessing evidence for nursing practice. $9^{\text {th }}$ ed. Philadelphia: Lippincott Williams and Wilkins; 2012

30. Kwon KH, Son BH, Han WK. Laparoscopic totally extraperitoneal repair without suprapubic port: comparison with conventional totally extraperitoneal repair. Journal of the Korean Surgical Society 2011;80(5):319-326.

31. Tai HC, Lin CD, Chung SD, et al. A comparative study of standard versus laparoendoscopic single-site surgery (LESS) totally extraperitoneal (TEP) inguinal hernia repair. Surgical Endoscopy. 2011;25(9):2879-83.

32. Kim JH, Lee YS, Kim JJ, et al. Single port laparoscopic totally extraperitoneal hernioplasty: a comparative study of short-term outcome with conventional laparoscopic totally extraperitoneal hernioplasty. World J Surg. 2013;37(4):746-751.

33. Tsai YC, Ho $\mathrm{CH}$, Tai $\mathrm{HC}$, et al. Laparoendoscopic single-site versus conventional laparoscopic total extraperitoneal hernia repair: a prospective randomized clinical trial. Surg Endosc. 2013;27(12):4684-4692.

34. Froylich D, Haskins IN, Aminian A, et al. Laparoscopic versus open inguinal hernia repair in patients with obesity: An American College of Surgeons NSQIP clinical outcomes analysis. Surg Endosc. 2016;31(3):1305-10

35. Carter J, Duh QY. Laparoscopic repair of inguinal hernias. World J Surg 2011;35(7):1519-25.

36. Laparoscopic surgery for inguinal hernia repair: 1-guidance: Guidance and guidelines. NICE; 2004.

37. Moher D, Schulz KF, Altman DG. The CONSORT statement: revised recommendations for improving the quality of reports of parallel-group randomised trials. Lancet. 2001;357(9263):1191-4.

38. Greenhalgh T. How to read a paper: the basics of evidence-based medicine. $5^{\text {th }}$ ed. New York: Wiley-Blackwell; 2014.

39. Weiss HG, Brunner W, Biebl MO, et al. Wound complications in 1145 consecutive transumbilical single-incision laparoscopic procedures. Ann Surg. 2014;259(1):89-95.

40. Booth CM, Tannock IF. Randomised controlled trials and populationbased observational research: partners in the evolution of medical evidence. Br J Cancer. 2014;110(3):551-5.

41. Krell RW, Birkmeyer NJ, Reames BN, et al. Effects of resident involvement on complication rates after laparoscopic gastric bypass. $J$ Am Coll Surg. 2014;218(2):253-260.

42. Johansen A, Romundstad L, Nielsen CS, et al. Persistent postsurgical pain in a general population: prevalence and predictors in the Troms $\varnothing$ study. Pain. 2012;153(7):1390-6

43. Zheng M, Qin M, Zhao $\mathrm{H}$, et al. Laparoendoscopic single-site cholecystectomy: a randomized controlled study. Minim Invasive Ther Allied Technol. 2012;21(2):113-117.

44. Aprea G, Coppola Bottazzi E, Guida F, et al. Laparoendoscopic single site (LESS) versus classic video-laparoscopic cholecystectomy: a randomized prospective study. J Surg Res. 2011;166(2):109-12.

Citation: Binbosayes AA. Comparison between laparoendoscopic single-site surgery and the conventional total extraperitoneal technique in adult patients for inguinal hernia repair concerning postoperative pain, complications, and cosmesis:A literature review. MOJ Surg. 20I8;6(4):99-II2. 
45. Sharma A, Soni V, Baijal M, et al. Single port versus multiple port laparoscopic cholecystectomy - a comparative study. Indian $J$ Surg. 2013;75(2):115-122.

46. Buckley FP, Vassaur H, Monsivais S, et al. Comparison of outcomes for single-incision laparoscopic inguinal herniorrhaphy and traditional three-port laparoscopic herniorrhaphy at a single institution. Surg Endosc. 2014;28(1):30-35.

47. Di Censo A. Evidence-based nursing: A guide to clinical practice. Elsevier Health Sciences; 2014.

48. Bailey S, Handu D. Introduction to epidemiologic research methods in public health practice. Burlington: Jones and Bartlett Publishers; 2012.

49. Merrill RM. Introduction to epidemiology. Jones and Bartlett Publishers; 2015 .

50. Sedgwick P. Retrospective cohort studies: advantages and disadvantages. BMJ. 2014;348(241):1072-1073.

51. Friberg L, Benson L, Rosenqvist M, et al. Assessment of female sex as a risk factor in atrial fibrillation in Sweden: nationwide retrospective cohort study. BMJ. 2012;344:3522

52. Privitera GJ. Research methods for the behavioral sciences. London: Sage Publications; 2013.

53. Shin J, Hartnick C, Randolph G. Evidence-based otolaryngology NewYork: Springer Science and Business Media; 2009.

54. Soon Y, Yip E, Onida S, et al. Single-port hernia repair: a prospective cohort of 102 patients. Hernia. 2012;16(4):393-396.

55. Ma J, Cassera MA, Spaun GO. et al. Randomized controlled trial comparing single-port laparoscopic cholecystectomy and four-port laparoscopic cholecystectomy. Annals of Surgery 2011;254(1):22-27.

56. St Peter SD, Adibe OO, Juang D, et al. Single incision versus standard 3-port laparoscopic appendectomy: a prospective randomized trial. Ann Surg. 2011;254(4):586-590

57. Kulacoglu H. Current options in inguinal hernia repair in adult patients. Hippokratia. 2011;15(3):223-231.

58. Rao A, Kynaston J, MacDonald ER, et al. Patient preferences for surgical techniques: should we invest in new approaches? Surgical Endoscopy. 2010;24(12):3016-3025.

59. Filipovic-Cugura J, Kirac I, Kulis T, et al. Single-incision laparoscopic surgery (SILS) for totally extraperitoneal (TEP) inguinal hernia repair: first case. Surg Endosc. 2009;23(4):920-21.

60. Swartz MH. Textbook of physical diagnosis: history and examination. $7^{\text {th }}$ ed. NewYork: Elsevier Health Sciences; 2014

61. Neligan PC. Plastic Surgery E-Book: 6-Volume Set: Expert ConsultOnline. Seattle: Elsevier Health Sciences; 2012.

62. Sim J, Wright C. Research in health care: concepts, designs and methods. Nelson Thornes; 2000.

63. Ahmed I, Paraskeva P. A clinical review of single-incision laparoscopic surgery. Surgeon. 2011;9(6):341-51

64. Do M, Liatsikos E, Beatty J, et al. Laparoendoscopic single-site extraperitoneal inguinal hernia repair: initial experience in 10 patients. $J$ Endourol. 2011;25(6):963-8.

65. de Araújo FB, Starling ES, Maricevich M, et al. Single site and conventional totally extraperitoneal techniques for uncomplicated inguinal hernia repair: A comparative study. J Minim Access Surg. 2014;10(4):197-201.

66. Teoh AY, Chiu PW, Wong TC, et al. A double-blinded randomized controlled trial of laparoendoscopic single-site access versus conventional 3-port appendectomy. Ann Surg. 2012;256(6):909-914.

67. Chung JH, Baek JM, Chung K, et al. A comparison of postoperative pain after transumbilical single-port access and conventional three-port total laparoscopic hysterectomy: a randomized controlled trial. Acta Obstet Gynecol Scand. 2015;94(12):1290-96.

68. Klaus A, Mühlmann GM, Laimer E. Single-incision laparoscopic surgery: Hernia repair. Asian Journal of Endoscopic Surgery. 2011;4(2):59-62.

69. Siddiqui MR, Kovzel M, Brennan SJ, et al. The role of the laparoendoscopic single site totally extraperitoneal approach to inguinal hernia repairs: a review and meta-analysis of the literature. Can $J$ Surg. 2014;57(2):116-26.

70. Yussra Y, Sutton PA, Kosai NR, et al. Single incision laparoscopic surgery (SILS) inguinal hernia repair-recent clinical experiences of this novel technique. Clin Ter. 2012;164(5):425-428.

71. Angioni S, Pontis A, Pisanu A, et al. Single-port access subtotal laparoscopic hysterectomy: a prospective case-control study. J Minim Invasive Gynecol. 2015;22(5):807-12.

72. Chen YJ, Wang PH, Ocampo EJ, et al. Single-port compared with conventional laparoscopic-assisted vaginal hysterectomy: a randomized controlled trial. Obstet Gynecol. 2011;117(4):906-12.

73. Sato H, Shimada M, Kurita N, et al. The safety and usefulness of the single incision, transabdominal pre-peritoneal (TAPP) laparoscopic technique for inguinal hernia. J Med Invest. 2012;59(3-4):235-240.

74. Arezzo A, Scozzari G, Famiglietti F, et al. Is single-incision laparoscopic cholecystectomy safe? Results of a systematic review and metaanalysis. Surg Endosc. 2013;27(7):2293-304.

75. Gill RS, Shi X, Al-Adra DP, et al. Single-incision appendectomy is comparable to conventional laparoscopic appendectomy: a systematic review and pooled analysis. Surg Laparosc Endosc Percutan Tech. 2012;22(4):319-27.

76. Lv C, Wu S, Wu Y, et al. Single-incision laparoscopic versus traditional multiport laparoscopic colorectal surgery - a cumulative metaanalysis and systematic review. International Journal of Colorectal Disease. 2013;28(5):611-621.

77. Białecki JT, Wieloch MM, Kołomecki K. Single incision approach to totally extraperitoneal inguinal hernia repair. Wideochir Inne Tech Maloinwazyjne. 2014;9(12):201-6.

78. Critical Appraisal Skills Programme. 2017

79. Champagne BJ, Papaconstantinou HT, Parmar SS, et al. Single-incision versus standard multiport laparoscopic colectomy: a multicentre, casecontrolled comparison. Annals of Surgery. 2012;255(1):66-69.

80. Coran AG, Caldamone A, Adzick NS, et al. Pediatric surgery. $7^{\text {th }}$ ed Philadelphia: Elsevier Health Sciences; 2012.

81. Cugura JF, Kirac I, Kulis T, etal.Comparison of single incision laparoscopic totally extraperitoneal and laparoscopic totally extraperitoneal inguinal hernia repair: initial experience. J Endourol. 2012;26(1):63-66.

82. Feliu X, Clavería R, Besora $\mathrm{P}$, et al. Bilateral inguinal hernia repair: laparoscopic or open approach? Hernia. 2011;15(1):15-18.

83. Figure 1. Laparoscopyendourology. Greece: University of Patras; 2017.

84. Figure 2. Laparoscopy-endourology. Greece: University of Patras; 2017.

85. Figure 9. Single Incision Laparoscopic Surgery. 2017.

86. Gangl O, Hofer W, Tomaselli F, et al. Single incision laparoscopic cholecystectomy (SILC) versus laparoscopic cholecystectomy (LC) - a matched pair analysis. Langenbecks Arch Surg. 2011;396(6):819-24.

Citation: Binbosayes AA. Comparison between laparoendoscopic single-site surgery and the conventional total extraperitoneal technique in adult patients for inguinal hernia repair concerning postoperative pain, complications, and cosmesis:A literature review. MOJ Surg. 20I8;6(4):99-II2. 
87. Gerrish K, Lathlean J. The Research Process in Nursing. $7^{\text {th }}$ ed Chichester: John Wiley and Sons; 2015.

88. Greaves N, Nicholson J. Single incision laparoscopic surgery in general surgery: a review. Ann R Coll Surg Engl. 2011;93(6):437-40.

89. Lee WS, Choi ST, Lee JN, et al. Single-port laparoscopic appendectomy versus conventional laparoscopic appendectomy: a prospective randomized controlled study. Annals of Surgery. 2013;257(2):214-218.

90. Sberwinter DA. Transitioning to single-incision laparoscopic inguinal herniorrhaphy. JSLS. 2010;14(3):353-7.
91. Tsimoyiannis E1, Tsimogiannis KE, Gogos PG, et al. Different pain scores in single transumbilical incision laparoscopic cholecystectomy versus classic laparoscopic cholecystectomy: a randomized controlled trial. Surg Endosc. 2010;24(8):1842-1848.

92. Wauschkuhn CA, Schwarz J, Boekeler U, et al. Laparoscopic inguinal hernia repair: gold standard in bilateral hernia repair? Results of more than 2800 patients in comparison to literature. Surg Endosc. 2010;24(12):3026-3030.

Citation: Binbosayes AA. Comparison between laparoendoscopic single-site surgery and the conventional total extraperitoneal technique in adult patients for inguinal hernia repair concerning postoperative pain, complications, and cosmesis:A literature review. MOJ Surg. 20I8;6(4):99-II2. 